\title{
Mecanismos de Transparência Pública e Participação Social adotados pela Universidade Federal de Pelotas com vistas ao cumprimento dos requisitos de transparência previstos na LAI
}

\author{
Paulo de Almeida Afonso ${ }^{1}$, Henrique Avila Vianna ${ }^{1}$, Júlio C. B. Mattos $^{12}$ \\ ${ }^{1}$ Pró-Reitoria de Gestão da Informação e Comunicação - PROGIC \\ ${ }^{2}$ Centro de Desenvolvimento Tecnológico - CDTec \\ Universidade Federal de Pelotas - UFPEL \\ Pelotas - RS - Brasil \\ \{paulo.afonso,hvianna\}@ufpel.edu.br, julius@inf.ufpel.edu.br
}

\begin{abstract}
The aim of this paper is to present the methodology and the mechanisms used by the Pro-Rectory of Information and Communication Management in order to comply with the transparency requirements of LAI, Federal Decree 7,724 / 2012 and other regulations that impose on federal agencies the obligation to disclose information of interest the society. In addition to promoting the opening of data at the Federal University of Pelotas, with efficiency and quality, the actions taken out have provided greater visibility of the administrative and academic activities of the Institution, resulting in a reduction of requests for access to information.
\end{abstract}

Resumo. Este trabalho tem por objetivo apresentar a metodologia e os mecanismos adotados pela Pró-Reitoria de Gestão da Informação e Comunicação com vistas ao cumprimento dos requisitos de transparência previstos na Lei de Acesso a Informação (LAI), no Decreto federal 7.724/2012 e em outros normativos que impõem aos órgãos públicos a obrigação de divulgar informações de interesse da sociedade. Além de promover a abertura de dados e transparência ativa da Universidade Federal de Pelotas, com eficiência e qualidade, as ações realizadas tem proporcionado maior visibilidade das atividades administrativas e acadêmicas da Instituição, resultando em uma redução dos pedidos de acesso à informação.

\section{Introdução}

Com o propósito de aprimorar e simplificar a relação entre o Estado e o Cidadão, diferentes soluções de Governo Aberto, particularmente aquelas baseadas em tecnologia da informação, tem sido utilizadas por governos nacionais e estrangeiros [Cynthia de Freitas et al. 2014]. Dessa forma, diversas iniciativas têm sido implementadas com o objetivo de simplificar serviços, agilizar processos e ampliar a transparência e a participação social. Essas iniciativas normalmente envolvem o provimento de dados reutilizáveis como uma entre uma série de etapas destinadas a aumentar a transparência geral dos governos.

A declaração multilateral do Governo Aberto, assinada pelo Brasil, Estados Unidos e seis outros países em setembro de 2011, prevê entre os acordos firmados, o comprometimento dos signatários no fornecimento de informações de alto valor 
[Yu and Robinson 2011]. Nesse contexto, destaca-se o provimento de dados brutos, em tempo hábil, e em formatos que o cidadão possa facilmente localizar, entender e reutilizar, situando essas novas tecnologias de compartilhamento de dados no contexto da responsabilidade política, com o reconhecimento de que pessoas em todo o mundo estão exigindo maior transparência do governo.

Como um dos co-fundadores da Open Government Partnership ${ }^{1}$, o Brasil possui um forte compromisso em ampliar a transparência pública, prevenir e combater a corrupção e fomentar os ideais democráticos com a participação do cidadão na tomada de decisões, com vistas à melhoria dos serviços públicos. Frente a este cenário, várias iniciativas tem sido desenvolvidas para melhorar sua estrutura legal, promover a participação dos cidadãos e usar a tecnologia para uma maior abertura das informações públicas disponíveis [OGP ]. Os compromissos assumidos pelo Brasil em seu primeiro Plano de Ação produziram diversos benefícios importantes, entre os quais podemos destacar:

- a criação do Portal Brasileiro de Dados Abertos², em dezembro de 2010;

- a organização da Conferência Nacional sobre Transparência e Controle Social ${ }^{3}$ e;

- a implementação da Lei $\mathrm{n}^{0} 12.527 / 2011^{4}$, denominada Lei de Acesso à Informação (LAI).

A LAI entrou em vigor em 16 de maio de 2012 e regulamenta o direito constitucional de acesso às informações públicas, estabelecendo mecanismos que possibilitam, a qualquer pessoa, física ou jurídica, sem necessidade de apresentar motivo, o recebimento de informações públicas dos órgãos e entidades. O Decreto $n^{0} 7.724$, de 16/05/2012 ${ }^{5}$, que a regulamenta, determina que cabe aos órgãos e entidades públicas assegurar o direito de acesso à informação, independente de requerimentos, promovendo sua divulgação em local de fácil acesso, mediante o uso de linguagem cidadã, a fim de tornar as informações claras e acessíveis.

O Plano de Ação da INDA (Infraestrutura Nacional de Dados Abertos) [INDA ] é o instrumento de planejamento no âmbito da política de dados abertos para atingir os objetivos da INDA em direção à sua visão institucional. Cabe a cada organização pública implementar essa política por meio de outro instrumento denominado Plano de Dados Abertos (PDA). O PDA deve ser elaborado pela própria instituição, mediante a definição de iniciativas específicas, de acordo com os procedimentos apresentados no Kit de Dados Abertos $^{6}$.

Além do Plano de dados abertos, para atendimento às demandas de acesso à informação de forma eficiente, faz-se necessário constituir mecanismos adequados para publicação dos dados a serem disponibilizados em formato aberto, de acordo com sua natureza e público alvo. Nesse contexto, o presente trabalho tem por objetivo apresentar a metodologia e os mecanismos adotados pela Pró-Reitoria de Gestão da Informação e Comunicação (PROGIC), com vistas ao cumprimento dos requisitos de transparência, na Universidade Federal de Pelotas (UFPEL). Duas ações se destacam neste cenário: a

\footnotetext{
${ }^{1}$ https://www.opengovpartnership.org/

${ }^{2} \mathrm{http}: / /$ dados.gov.br/

${ }^{3}$ http://www.cgu.gov.br/assuntos/controle-social/consocial

${ }^{4}$ http://www.planalto.gov.br/ccivil_03/_ato2011-2014/2011/lei/112527.htm

${ }^{5}$ http://www.planalto.gov.br/ccivil_03/_ato2011-2014/2012/Decreto/D7724.htm

${ }^{6} \mathrm{http}: / /$ kit.dados.gov.br/
} 
implantação do Portal de Dados Abertos da UFPel ${ }^{7}$ e o Portal Institucional da UFPel ${ }^{8}$. As ações realizadas, além de atenderem a legislação vigente, proporcionaram a ampliação e o aprimoramento da abertura de dados sem restrição de acesso, zelando pelos princípios da publicidade, transparência e eficiência. Além disso, por meio da disseminação de dados e informações para a sociedade em formatos reutilizáveis, espera-se incentivar a participação social e promover a melhoria da qualidade dos dados disponibilizados.

O artigo está organizado da seguinte maneira: a seção 2 apresenta os trabalhos relacionados; na seção 3 é descrita a abordagem utilizada e os mecanismos implementados para promoção da transparência na instituição; na seção 4 são apresentados os resultados obtidos e, por fim, na seção 5 são apresentadas as conclusões.

\section{Trabalhos relacionados}

Com o objetivo de promover a transparência pública, em atendimento à legislação vigente, diferentes abordagens tem sido propostas para assegurar a disponibilização de dados em formato aberto e acessível pelo cidadão. Tais soluções preveem a integração de plataformas online, participação social e publicação dinâmica de informações.

Em [de França et al. 2018], é apresentado um estudo com base na extração de dados relacionados a duas iniciativas voltadas a propagação da cultura de transparência na Universidade Estadual de Campinas, a saber: um Curso de transparência de dados e um Hackathon de transparência de dados. A partir da análise dos dados coletados, os autores apresentaram uma arquitetura de software como uma infraestrutura para apoiar a disponibilização e acesso aos dados públicos da instituição, de forma facilitada e irrestrita.

O trabalho apresentado por [Pranto Filho et al. 2018] aborda a estratégia e a metodologia utilizada para a construção do Plano de Dados Abertos e a publicação dos dados abertos do Tribunal de Contas do Rio Grande do Norte (TCE/RN), a partir de uma parceria acadêmica com o Instituto Metrópole Digital (IMD), uma Unidade Acadêmica Especializada da Universidade Federal do Rio Grande do Norte.

Em [Biffi et al. 2017] é proposto um método que aborda a publicação dinâmica de informações democráticas e busca a possibilidade de diálogo com o ambiente externo à organização, com vistas ao alcance de melhores níveis de maturidade em transparência organizacional. O método denominado TRADIN, integra múltiplas fontes de sistemas de informações internas dos órgãos de governo, proporcionando o uso destas por outros órgãos governamentais ou outros segmentos da sociedade, de forma integrada e transparente.

De forma análoga às abordagens anteriormente citadas, a participação social e a integração entre sistemas foram consideradas estratégias eficientes para análise e escolha dos dados a serem disponibilizados pela UFPEL. A seção a seguir apresenta a metodologia adotada, assim como os mecanismos e tecnologias utilizados.

\footnotetext{
${ }^{7}$ http://dados.ufpel.edu.br/

${ }^{8}$ https://institucional.ufpel.edu.br/
} 


\section{Metodologia}

A metodologia empregada para abertura de dados na UFPEL teve início com uma revisão bibliográfica, tomando-se por base as instruções e normativos relacionados ao tema e, considerando o contexto de acesso à informação, o grau de relevância para o cidadão. Para a realização deste trabalho foi constituída uma comissão, composta por nove servidores de diferentes áreas: Comunicação Social, Ouvidoria, Tecnologia da Informação e Informações Institucionais. Além dos normativos, a Comissão considerou em sua abordagem inicial os seguintes elementos:

1. As demandas recebidas através do Sistema Eletrônico do Serviço de Informações ao Cidadão (e-SIC);

2. Solicitações de informação recebidas através de email;

3. Informações relacionadas aos setores e serviços mais procurados nos sítios eletrônicos da IES;

4. O alinhamento perante o Planejamento Estratégico e os relacionados às áreas de tecnologia da informação;

5. O conjunto de informações e sistemas sob a gestão da IES, em especial os sistemas estruturadores que são de uso obrigatório transversal para os órgãos da administração pública; e

6. O nível de maturidade da organização das informações e dados existentes.

O trabalho da comissão resultou na elaboração do Plano de Dados Abertos ${ }^{9}$ (PDA) da UFPEL. Um documento orientador para as ações de implementação e promoção de abertura de dados na instituição, mediante a observação de padrões mínimos de qualidade, de forma a facilitar o entendimento e a reutilização das informações.

O PDA organiza a criação e a implantação racional dos processos de publicação e sustentabilidade de dados, bem como traz as referências da literatura e dos materiais disponíveis sobre o ferramental técnico. A primeira versão do PDA/UFPEL, apresenta um conjunto de dados disponível em transparência ativa, acessível através do Portal de dados Abertos da UFPEL ${ }^{10}$.

Além do Portal de Dados Abertos, se identificou uma série de falhas na divulgação de informações institucionais na UFPel, principalmente em relação a cursos, projetos, servidores, unidades entre outros. Desta forma, constitui-se uma comissão com membros da PROGIC e comunidade acadêmica que elaborasse uma proposta que permitisse um portal com layout padronizado das páginas possibilitando ao visitante familiarizar-se mais rapidamente com o Portal e que assegurasse que o mesmo conjunto de informações estivesse disponível e facilmente localizável em todas as consultas. Além disso, as páginas do Portal deveriam ser geradas dinamicamente das bases de dados do sistema de gestão da instituição (COBALTO). Atualmente, o Portal Institucional é um dos principais mecanismos facilmente acessíveis de transparência ativa.

\subsection{Portal de Dados Abertos da UFPEL}

O Portal de Dados Abertos da UFPEL (http://dados.ufpel.edu.br/) objetiva promover a abertura de dados na Instituição, atendendo a padrões mínimos de qualidade, de forma a

\footnotetext{
${ }^{9} \mathrm{https}: / /$ wp.ufpel.edu.br/acessoainformacao/files/2018/04/PDA-UFPEL-2018-2019.pdf

${ }^{10} \mathrm{http}: / /$ dados.ufpel.edu.br/
} 
facilitar o entendimento e a reutilização das informações. Através do Portal são disponibilizados para toda a comunidade dados e informações públicas, que podem ser usadas no desenvolvimento de aplicativos e ações. A abertura de dados objetiva, sobretudo, garantir os princípios da publicidade e da transparência da administração pública, devendo ser considerados os seguintes aspectos [Eaves 2009]:

- Se o dado não pode ser encontrado e indexado na web, ele não existe;

- Se não estiver aberto e disponível em formato compreensível por máquina, ele não pode ser reaproveitado;

- Se algum dispositivo legal não permitir sua replicação, ele não é útil.

Considerando tais características, a solução adotada para a publicação dos dados foi o CKAN - Comprehensive Knowledge Archive Network ${ }^{11}$, uma ferramenta de código aberto para gerenciamento e publicação de dados, bastante utilizada por governos nacionais e internacionais, instituições de pesquisa e outras organizações que lidam com grande quantidade e diversidade de dados. O CKAN permite a integração do Portal de Dados Abertos da UFPEL ao Portal Brasileiro de Dados Abertos (http://dados.gov.br/dataset?q=ufpel), atendendo às mais recentes normas da legislação vigente.

Atualmente são disponibilizados através do Portal de Dados Abertos 27 conjuntos de dados relacionados à graduação, pós-graduação, contratação de serviços, orçamento, bolsas, manifestações do e-OUV, solicitações via e-SIC, entre outros. A Figura 1 apresenta a página inicial do portal de dados abertos.

\subsection{Participação Social}

A Resolução CGINDA n ${ }^{\circ} 3 / 17$ determina, em seu art. $1^{\circ}, \S 1^{\circ}$, a utilização obrigatória de mecanismo de participação social como: audiência pública, consulta pública na internet ou outra estratégia de interação com a sociedade. Dessa forma, foi aberto um canal de comunicação com a sociedade para consultar se existem outras bases de dados que gostariam que fossem disponibilizadas na vigência do PDA. Por meio de um formulário eletrônico específico (https://wp.ufpel. edu.br/acessoainformacao/consultapublica-ufpel-dados-abertos/) e mediante consulta ao inventário de base de dados da UFPEL, o cidadão pode contribuir para a priorização e abertura de dados da instituição.

\subsection{Portal Institucional}

O Portal Institucional UFPEL ${ }^{12}$ disponibiliza informações acadêmicas e administrativas de forma simples e estruturada, colocando à disposição da comunidade dados consolidados de 298 cursos, 5.139 disciplinas, 7.378 projetos, 458 unidades e 2.795 servidores.

A proposta nasceu a partir de um diagnóstico no qual se identificou uma série de falhas na divulgação de informações institucionais na UFPel, entre as quais podemos destacar: (i) nem todas as informações exigidas pela legislação estavam disponíveis ao público; (ii) informações importantes para a comunidade acadêmica estavam pulverizadas entre diversos sites de cursos e unidades, com diferentes formas de apresentação, dificultando sua localização; (iii) as informações disponíveis precisavam ser atualizadas manualmente em diversos locais, ocasionando atrasos e inconsistências.

\footnotetext{
${ }^{11} \mathrm{https}: / / \mathrm{ckan}$. org/

${ }^{12} \mathrm{https} / / /$ institucional.ufpel.edu.br/
} 


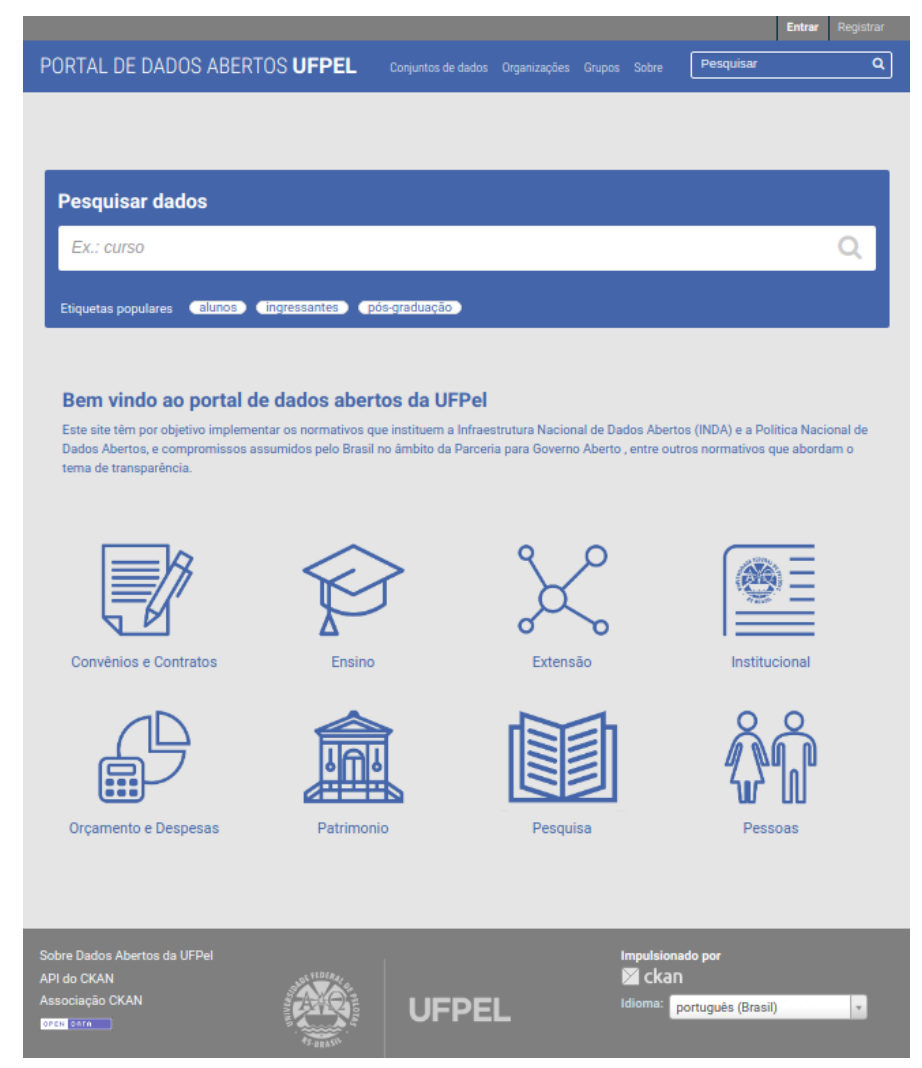

Figura 1. Portal de Dados Abertos UFPEL.

Havia também uma preocupação em divulgar ao público em geral o máximo possível de informações, que até então estavam restritas a sistemas internos da Universidade. Com essa visão, foi elaborada a proposta de um portal agregador de informações, com os seguintes objetivos:

- Contemplar as exigências da legislação na divulgação de informações institucionais;

- Agregar outras informações de interesse da comunidade acadêmica e do público em geral;

- Facilitar o acesso à informação na UFPel;

- Garantir a consistência, fidelidade e atualização das informações apresentadas.

O layout padronizado das páginas possibilita ao visitante familiarizar-se mais rapidamente com o Portal e assegura que o mesmo conjunto de informações esteja disponível e facilmente localizável em todas as consultas. Gestores de cursos e unidades podem adicionar, também, links para suas redes sociais e websites próprios, onde podem disponibilizar conteúdos complementares.

As páginas do Portal são geradas dinamicamente, com dados obtidos em tempo real do sistema integrado de gestão da UFPEL (COBALTO), que engloba os processos acadêmicos e administrativos da Universidade. Dessa forma, garante-se a divulgação de informações sempre atualizadas, sem demandar esforço adicional por parte das unidades.

Todas as informações disponíveis no Portal estão dinamicamente conectadas - a partir de uma consulta inicial é possível navegar por milhares de páginas de conteúdo, 
através de referências cruzadas, tornando-se também uma excelente ferramenta de descoberta da Universidade. A Figura 2 apresenta as interconexões entre os objetos do portal.

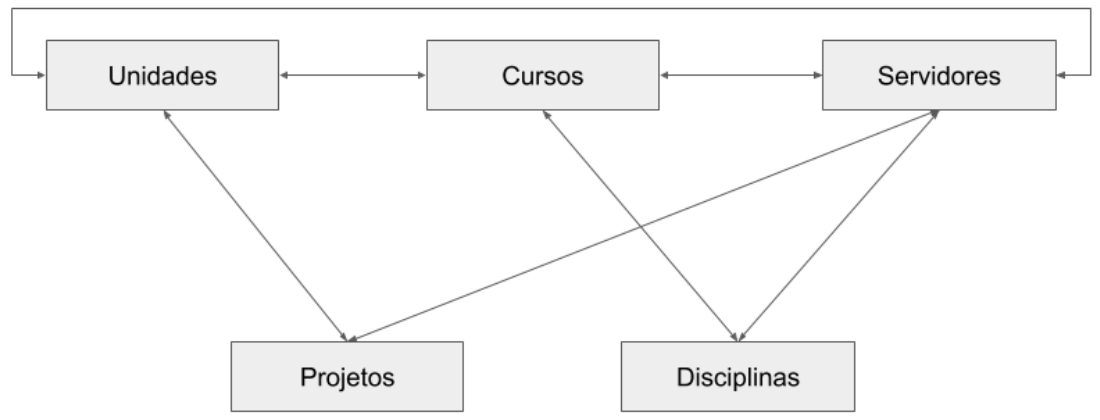

Figura 2. Diagrama de interconexão entre objetos no Portal Institucional.

Para maior agilidade na localização das informações, o Portal oferece ainda pesquisa personalizada, utilizando Elasticsearch - um mecanismo de busca orientado a documentos e otimizado para buscas textuais em grandes volumes de dados. O Portal atende às mais recentes recomendações de acessibilidade digital e adapta-se a diferentes tamanhos de tela, proporcionando excelente visualização também em dispositivos móveis. A Figura 3 apresenta a página inicial do Portal Institucional.

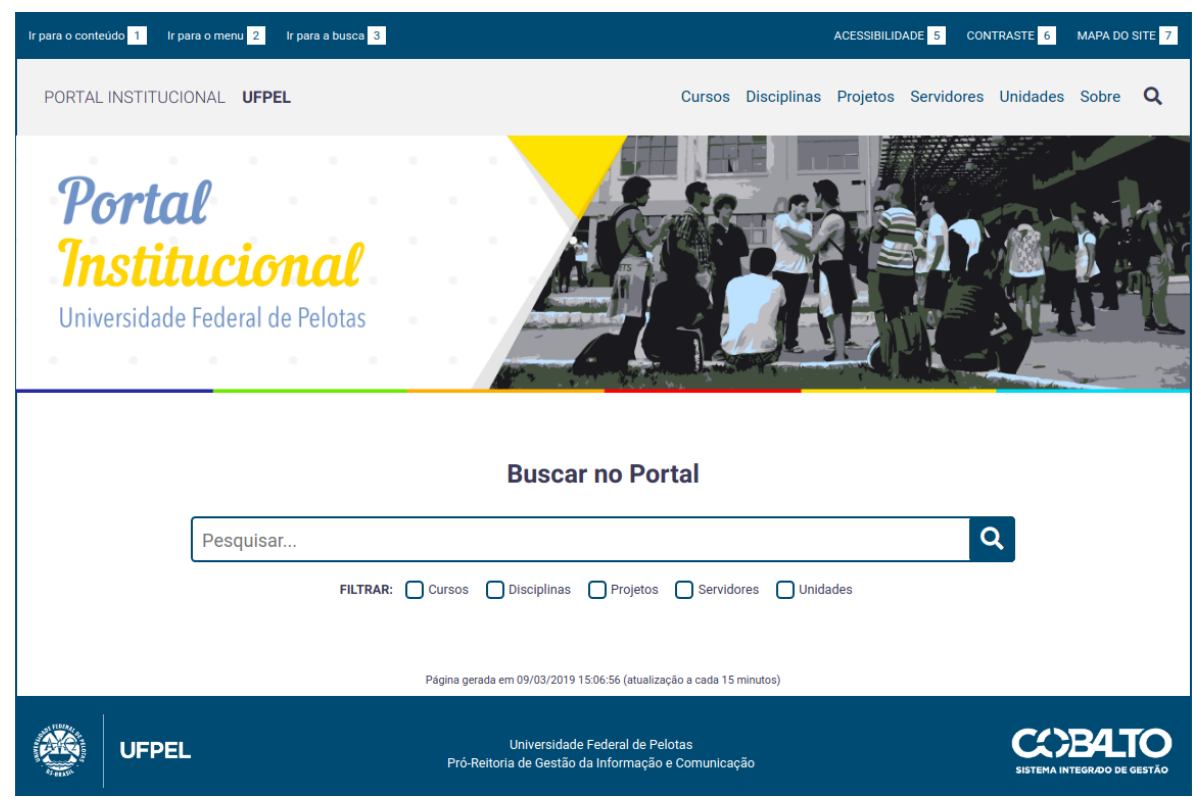

Figura 3. Portal Institucional UFPEL.

\section{Resultados}

A implantação do Portal de Dados Abertos e do Portal Institucional resultou em uma redução significativa dos pedidos de acesso a informação na UFPEL. Através do Portal de Dados Abertos, são atendidas exigências legais para publicação de dados de diferentes 
setores da instituição, facilitando o trabalho e proporcionando o atendimento aos órgãos de controle e ao cidadão de forma eficaz e com qualidade.

O gráfico da Figura 4 demonstra que o número de pedidos de acesso à informação teve uma pequena redução em 2018, o que não ocorria nos últimos 4 anos, anteriores à implantação do Portal de Dados Abertos, período em que o número de pedidos de acesso a informação, em relação à 2012, sofreu um aumento em torno de $65 \%$.

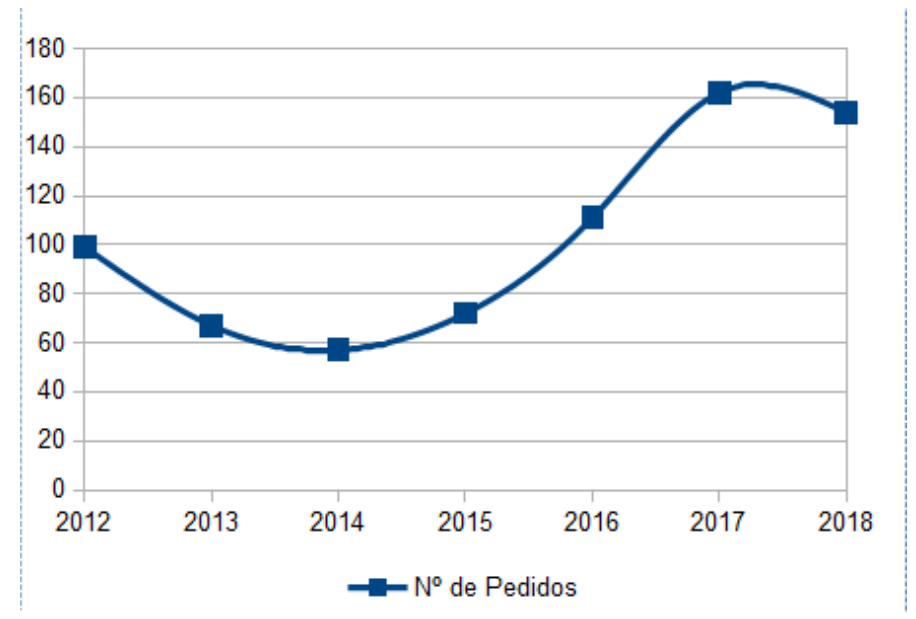

Figura 4. Pedidos de acesso a informação recebidos anualmente via e-SIC

A análise do primeiro trimestre de cada ano (Figura 5) demonstra uma queda significativa nos pedidos de acesso à informação em 2019. Um resultado bastante positivo, resultante da divulgação de informações atualizadas e em transparência ativa.

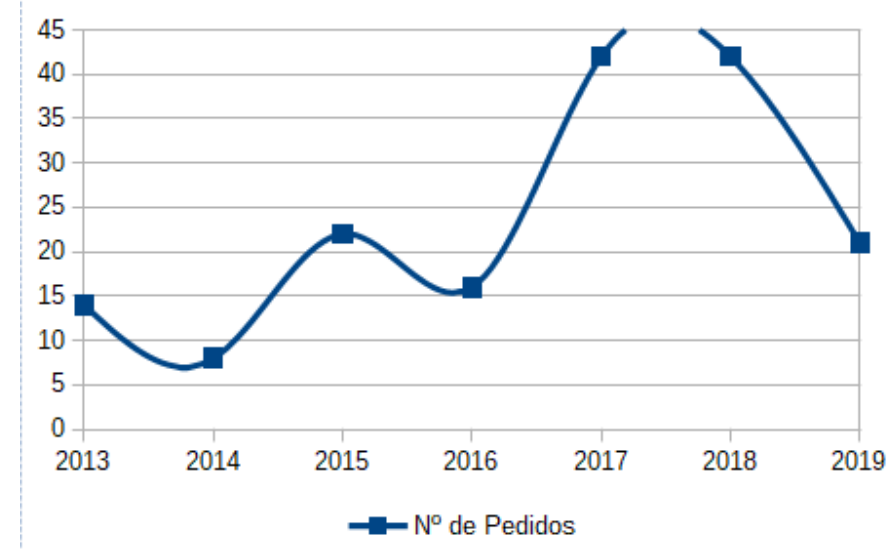

Figura 5. Pedidos de acesso a informação recebidos no primeiro trimestre de cada ano através do e-SIC

Desde julho de 2017, quando o Portal Insitucional foi disponibilizado ao público, até janeiro de 2019, o Portal Institucional registrou em média mais de 14 mil visitantes únicos por mês e mais de 80 mil visualizações de páginas por mês conforme apresentado na Figura 6. 


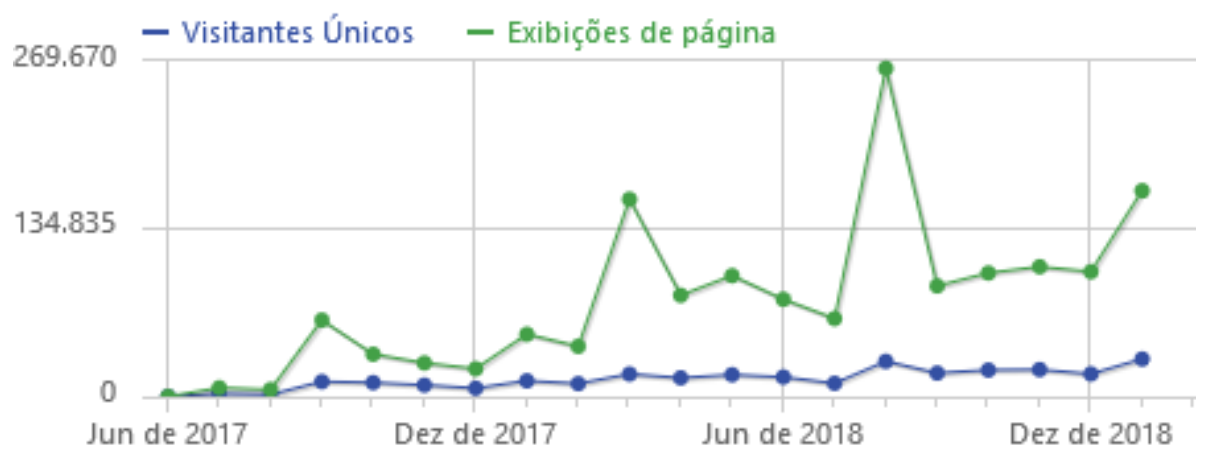

Figura 6. Acessos ao Portal Institucional.

\section{Conclusão}

O cenário atual apresenta-se como uma mudança de paradigma, voltado aos princípios de publicidade e transparência, constituindo a publicidade e a cultura do acesso como regra e o sigilo, como exceção [Hoch et al. 2012]. Percebe-se que a disponibilização de dados em transparência ativa, além de atender aos órgãos de controle, pode contribuir para a eliminação do retrabalho na prestação de informações institucionais para a sociedade, prestando um serviço de qualidade, com dinamismo e criatividade. Não basta, entretanto, realizar a publicação de informações de forma indiscriminada. Sua disponibilização deve observar limitações de privacidade, segurança e controle de acesso. Além disso, deve possibilitar a gravação de relatórios em formatos eletrônicos abertos e não proprietários, de modo a facilitar a análise das informações. Nesse contexto, destaca-se a importância da utilização das Tecnologias de Informação e de Comunicação, como alternativas eficazes para promover a abertura de dados e a comunicação e interação com a sociedade.

\section{Referências}

Biffi, G. C., Nunes, E. P. S., and Maciel, C. (2017). Método tradin: transparência governamental com a cooperação da economia digital. In $5^{\circ}$ Workshop de Transparência em Sistemas (WTranS), volume 5. SBC.

Cynthia de Freitas, Q. B., de Mello, P. J. S. M., and Camargo, R. M. P. (2014). Governo aberto: a tecnologia contribuindo para maior aproximação entre o estado e a sociedade. Revista do TCU, (131):30-39.

de França, B. B. N., dos Reis, J. C., and de Azevedo, R. J. (2018). Desafios sociotécnicos e prospecções para promover transparência de dados na universidade. In VI Workshop de Transparê ncia em Sistemas (WTranS), volume 6. SBC.

Eaves, D. (2009). The three laws of open government data. Eaves. ca, 30:8.

Hoch, P. A., Rigui, L. M., and da Silva, R. L. (2012). Desafios à concretização da transparência ativa na internet, à luz da lei de acesso à informação pública: análise dos portais dos tribunais regionais federais. Revista direitos emergentes na sociedade global, 1(2):257-286.

INDA. Infraestrutura nacional de dados abertos. Disponível em: http://wiki. dados.gov.br/Plano-de-Acao-da-INDA-2017-2018.ashx. Acesso em: 20-03-2019. 
OGP. Open government partnership. Disponível em: https://www . opengovpartnership.org/countries/brazil. Acesso em: 20-03-2019.

Pranto Filho, F. N., Oliveira, R., Pereira, L. S., and Ramalho, J. (2018). A iniciativa da construção do plano de dados abertos do tribunal de contas do rio grande do norte através de parceria acadêmica com a universidade federal do rio grande do norte. In VI Workshop de Transparê ncia em Sistemas (WTranS), volume 6. SBC.

Yu, H. and Robinson, D. G. (2011). The new ambiguity of open government. UCLA L. Rev. Discourse, 59:178. 